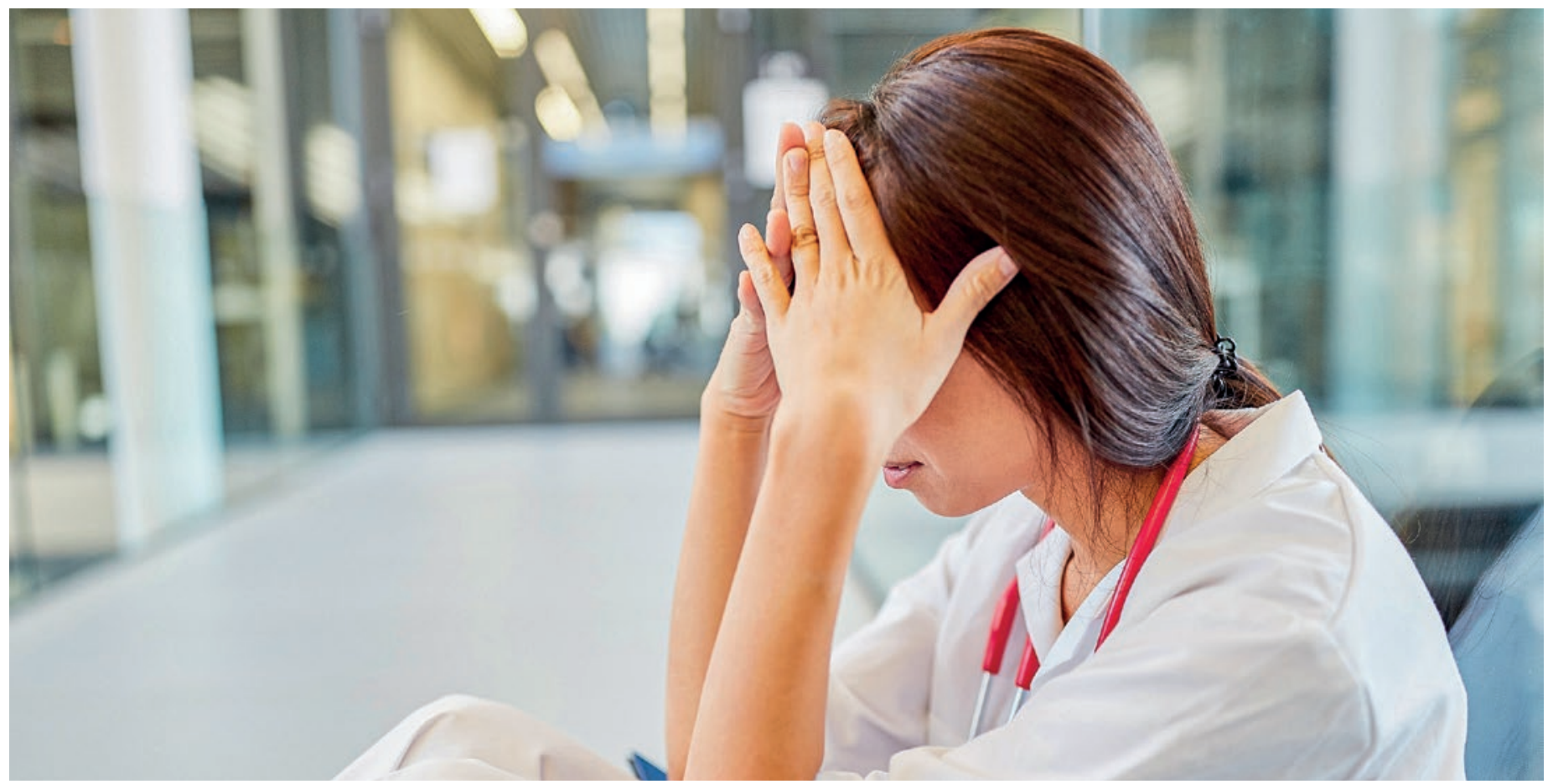

\title{
Die Angst vor der Fehldiagnose
}

\section{Patrick Stacha, Adrian Vogler}

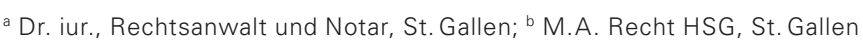

\author{
Zeit- und Kostendruck, Müdigkeit, Unachtsamkeit oder schlicht fehlendes Wissen - \\ die Gründe für eine Fehldiagnose sind vielfältig. In welchen Fällen aber haftet die \\ Ärztin, der Arzt für eine fehlerhafte Diagnose?
}

Ein Patient begibt sich mit starken Kopfschmerzen, Übelkeit, Erbrechen und Bauchschmerzen im epigastrischen Bereich in die Notaufnahme des Kantonsspitals Freiburg. Der diensthabende Assistenzarzt führt eine neurologische Untersuchung durch, bei der auch nach

Ein solches Horrorszenario hat Folgen für den Patienten und belastet den Arzt, der die Fehldiagnose stellte.

Anzeichen einer Hirnhautentzündung gesucht wird. In der Folge kommt er zum Schluss, dass es sich lediglich um eine Grippe handelt, und verabreicht dem Patienten Schmerzmittel. Schliesslich schickt er den Patienten auf dessen Wunsch nach Hause. Drei Stunden später wird der Patient wieder ins Krankenhaus eingeliefert, wo eine Pneumokokken-Meningitis festgestellt und mit Antibiotika behandelt wird. Der Krankheitsverlauf ist durch schwere neurologische und kardiovaskuläre Komplikationen gekennzeichnet, die eine langwierige Reanimation erfordern. Der Patient erwacht sechs Tage später aus dem Koma und ist beidseitig, aufgrund einer Schädigung der Innenohren, total taub [1].

Ein solches Horrorszenario zieht nicht nur Folgen für den Patienten mit sich, sondern belastet auch den (Assistenz-)Arzt, der die Fehldiagnose stellte. Nachfolgend sollen jedoch nicht die physischen und psychischen Folgen eines Diagnosefehlers beleuchtet werden, sondern die haftpflichtrechtlichen.

\section{Sorgfaltspflichtverletzung}

Das Bundesgericht formuliert die Sorgfaltspflicht des Mediziners wie folgt: «Die Besonderheit der ärztlichen Kunst liegt darin, dass der Arzt mit seinem Wissen und Können auf einen erwünschten Erfolg hinzuwirken 
hat, was jedoch nicht heisst, dass er diesen auch herbeiführen oder gar garantieren müsse» [2]. Wesentlich ist folglich, dass der Behandelnde nach den Regeln der ärztlichen Kunst (lege artis) handelt.

Der Arzt ist deshalb nicht dazu verpflichtet, eine korrekte Diagnose zu erstellen. Er ist lediglich dazu verpflichtet, seine Diagnose auf Ergebnisse zu stützen, die lege artis erhoben wurden. Dies bedeutet nicht, dass sämtliche zur Verfügung stehende Untersuchungsmethoden ausgeschöpft werden müssen, bevor eine Diagnose gestellt werden kann. Vielmehr muss der Mediziner sämtliche Untersuchungen vornehmen, die nach den Umständen geboten sind. Ergeben sich aufgrund der Untersuchungsergebnisse mehrdeutige Krankheitsbilder, muss der Arzt diese durch weitere Erhebungen aufklären.

Ein bundesgerichtlicher Entscheid aus dem Jahr 2003 [3] bejahte die Sorgfaltspflichtverletzung eines Notfallarztes, welcher, gestützt auf die wahrheitswidrigen Aussagen eines Unfallzeugen ein schweres SchädelHirn-Trauma übersah resp. nicht danach gesucht hatte. Der schwer alkoholisierte Patient liess sich von seinem Freund mit einem Motorroller bei $30 \mathrm{~km} / \mathrm{h}$ auf einem Rollbrett ziehen. Nach dem Sturz blieb der Patient regungslos auf der Strasse liegen. Dem behan-

\section{Nicht sämtliche Untersuchungsmethoden müssen ausgeschöpft werden, sondern jene, die nach den Umständen geboten sind.}

delnden Arzt berichtete der Freund, er habe den Patienten zu Fuss gestossen und dieser habe weder sein Bewusstsein verloren noch sei er mit dem Kopf auf dem Boden aufgeschlagen. Gemäss Formular 144 ergab die Prüfung des Bewusstseinszustands durch den Rettungsdienst einen Wert von 13 auf der Glasgow Coma Scale. Nach Dafürhalten des Bundesgerichts hat der verantwortliche Arzt nicht sämtliche gebotenen Untersuchungen am Patienten durchgeführt, da er sich nicht auf die Schilderungen des Freunds verlassen durfte, und sprach den Arzt der fahrlässigen schweren Körperverletzung schuldig.

\section{Die Regeln der wissenschaftlichen Kunst}

Das Bundesgericht anerkennt, dass die Beurteilung der Sorgfaltspflichtverletzung ex ante erfolgen muss. Ausschlaggebend ist somit nicht die nachträgliche Betrachtung der Behandlung, sondern die Überlegung, wie eine Durchschnittsperson derselben Berufsgattung zu jenem Zeitpunkt gehandelt hätte. Zu beachten sind Faktoren wie der zeitliche Entscheidungsdruck oder der Stand der Wissenschaft zum Zeitpunkt der Entscheidung.

Im Jahr 1938 [4] sprach das Bundesgericht einen Landarzt frei, welcher einem unter Alkoholeinfluss schwer erregten Passanten Beruhigungsmittel verabreichte, der in der Folge verstarb. Der Patient wurde nachts mit Hilfe von sieben Mann in das Sprechzimmer des Arztes verbracht. Dort wurden ihm unter anderem das opiumhaltige Pantopon, Chloralhydrat und Scopolamin verabreicht.

\section{Das Gericht stellte an den Landarzt nicht die gleichen Anforderungen wie an einen Spezialisten.}

Die Angehörigen des Patienten warfen dem Arzt später unter anderem vor, er habe bereits bei der Diagnose einen Fehler gemacht. Dem folgte das Gericht nicht und stützte das Urteil auf dem Expertenbefund, welcher auf einen pathologischen Rausch schloss. Interessant ist auch, dass das Gericht dem Landarzt nicht vorwarf, dass er neueste medizinische Kenntnisse nicht beachtet hatte, wonach die Einzelwirkung von Medikamenten potenziert wird, wenn sie verschiedene Angriffspunkte haben. Es anerkannte zwar die Pflicht des Arztes, sich stetig fortzubilden, stellte an den Landarzt jedoch nicht die gleichen Anforderungen wie an einen Spezialisten. Dies gilt nicht nur bei der Behandlung von Krankheitsbildern, sondern auch bei Diagnosen. Davon abzugrenzen ist die Pflicht, den Patienten an einen Spezialisten resp. ein Krankenhaus weiterzuverweisen, wenn die Gerätschaften oder das Know-how zur Stellung einer definitiven Diagnose fehlt. So ging das Bundesgericht nicht auf das Argument eines Hausarztes ein, welcher eine Aortendissektion nicht feststellen konnte, da ihm als Allgemeinpraktiker die In frastruktur zur Diagnose nicht zur Verfügung stehe. Vielmehr sei gemäss Bundesgericht zu überprüfen, ob nicht die unterlassene notfallmässige Einweisung in ein Spital eine Sorgfaltspflichtverletzung darstelle [5].

\section{Natürlicher und adäquater Kausalzusammenhang}

Ein einfacher Kausalzusammenhang zwischen der Sorgfaltspflichtverletzung und dem Schaden allein genügt nicht, um die Haftung des Arztes zu begründen. Vielmehr muss der Diagnosefehler nach dem gewöhnlichen Lauf der Dinge und der allgemeinen Lebenserfahrung dazu geeignet sein, den Schaden herbeizuführen [6]. Die Adäquanz des Kausalzusammenhangs beschränkt die ärztliche Haftpflicht auf Schadensfolgen, welche zum Zeitpunkt der Behandlung 
vorhersehbar waren. Da der medizinische Tätigkeitsbereich ohnehin gefahrenbehaftet ist, soll durch die Voraussetzung der Adäquanz die Haftung aufgrund von seltenen und unwahrscheinlichen Folgen ausgeschlossen werden.

So wurde der adäquate Kausalzusammenhang beispielsweise verneint, als ein Patient infolge einer Augenoperation zur Korrektur einer leichten Kurzsichtigkeit einen körperlichen Schaden in Form eines erheblichen Abfalls der Sehschärfe und der Wahrnehmung von Lichthöfen erlitt. Das ärztliche Gutachten kam zum Schluss, dass die Komplikationen, welche beim Patienten auftraten, so selten waren, dass kein vergleichbarer Fall in der Literatur gefunden werden konnte [7].

\section{Fehldiagnose oder Diagnosefehler?}

Nicht jede Fehldiagnose ist auch als Diagnosefehler zu werten und führt zur Arzthaftung. Ausschlaggebend in diesem Bereich ist, dass der behandelnde Arzt nach allen Regeln der Kunst gehandelt hat. Die Beurteilung der Handlung muss dabei aus dem Blickwinkel einer Durchschnittsperson derselben Berufsgattung zum Zeitpunkt des Diagnosefehlers erfolgen. Beachtet werden insbesondere der zeitliche Druck und der Stand der Wissenschaft zum Zeitpunkt der Diagnose. Es empfiehlt sich aus rechtlicher Sicht des-

\section{Das Wichtigste in Kürze}

- Wesentlich zur Beurteilung, ob es sich um einen Diagnosefehler handelt, ist die Frage, wie eine Durchschnittsperson derselben Berufsgattung unter denselben Umständen vorgegangen wäre.

- Der Arzt ist nicht verpflichtet, sämtliche Untersuchungsmethoden auszuschöpfen, sondern nur jene, die nach den Umständen geboten sind.

- Das Nichtvorhandensein von Gerätschaft und Fachwissen kann eine Verpflichtung begründen, den Patienten an einen geeigneten Spezialisten

oder das Spital zu überweisen. halb, trotz des hohen Arbeitspensums dort mit einer voreiligen Diagnose zuzuwarten, wo die Zeit nicht drängt. Obwohl einem Arzt nicht zugemutet werden kann, sämtliche ihm zur Verfügung stehenden Untersuchungsmethoden auszuschöpfen, erfordern

\section{Schliesslich sollte man im Zweifelsfall} nicht zögern, auch mal einen Kollegen zu konsultieren.

mehrdeutige Krankheitsbilder dennoch weitere $\mathrm{Ab}$ klärungen und wo diese nicht möglich sind, eine Einweisung in ein Spital. Es empfiehlt sich, die Diagnose auf eine breite Wissensbasis abzustützen und zu dokumentieren, aufgrund welcher symptome sie gestellt wurde. Schliesslich sollte man im Zweifelsfall nicht zögern, auch mal einen Kollegen zu konsultieren.

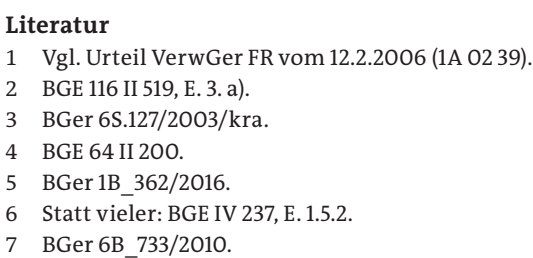

\section{L'essentiel en bref}

- Il est essentiel de savoir comment une personne moyenne de la même profession aurait procédé dans les mêmes circonstances pour évaluer s'il $s^{\prime}$ agit d'une erreur de diagnostic.

- Le médecin n'est pas tenu d'épuiser toutes les options d'examen, mais seulement celles exigées par les circonstances.

- L'absence d'équipement et d'expertise peut donner lieu à l'obligation d'adresser le patient à un spécialiste approprié ou à I'hôpital. 